\title{
A Rare Case of Intestinal Obstruction from Internal Hernia through a Foramen Formed by Appendices Epiploicae - A Case Report and Review of Literature
}

\author{
Thomas Olagboyega Olajide*, Olanrewaju Samuel Balogun \\ Department of Surgery, General Surgery Unit, College of Medicine, University of Lagos, Lagos University Teaching Hospital, \\ Lagos, Nigeria
}

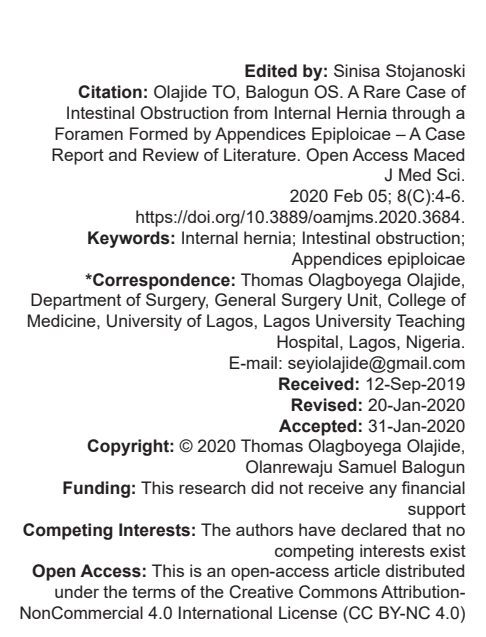

\author{
Abstract \\ BACKGROUND: Internal hernias are uncommon causes of acute abdomen and intestinal obstruction. Internal \\ herniation due to appendices epiploicae is very rare with only six cases reported in the literature. \\ CASE REPORT: We, herein, present the report of a 64-year-old female who presented with features of intestinal \\ obstruction due to internal herniation of a loop of small intestine through an orifice formed by the fusion of two \\ appendices epiploicae. The band was divided into release the entrapped loop of bowel. \\ CONCLUSION: A high index of suspicion with prompt surgical intervention will improve outcome.
}

\section{Introduction}

Internal hernia is an unusual cause of intestinal obstruction as post-operative adhesions and external hernias are the main etiology [1], [2]. This should be suspected when the patient with no history of the previous abdominal surgery and no clinical feature of obstructing external hernia or abdominal tumor develops intestinal obstruction. Herniation of the viscous may be through a normal anatomical aperture or an abnormal one within the peritoneal cavity. Internal hernia resulting from the appendices epiploicae is extremely rare with only six cases reported in the literature [3], [4], [5], [6], [7], [8].

\section{Case Report}

A 64-year-old female presented to the emergency department with a 5-day history of colicky abdominal pain, several episodes of bilious vomiting, and progressive abdominal distension, with obstipation starting a day later. There was no fever, weight loss, or previous abdominal surgery. There was no known comorbid factor. She had received medical care elsewhere intravenous fluids and some unknown medications were administered before referral. Physical examination revealed an acutely ill looking patient in mild respiratory distress (respiratory rate - 28 cycles/min), dehydrated, tachycardic (pulse rate $-144 / \mathrm{min}$ ), and afebrile. The chest was clinically clear. The abdomen was grossly distended and tense with no sign of peritonism, and bowel sound was hypoactive. Digital rectal examination was normal. A clinical diagnosis of intestinal obstruction secondary to the colonic tumor was made. She was resuscitated with intravenous fluid, nasogastric decompression, and oxygen through nasal prongs. Intravenous antibiotics were also commenced. Biochemistry revealed hypokalemia (serum potassium - $3.2 \mathrm{mmol} / \mathrm{l}$ ) with normal complete blood count. The abdominal computed tomography (CT) scan revealed distension of the small bowel and no mass lesion was observed (Figure 1). 


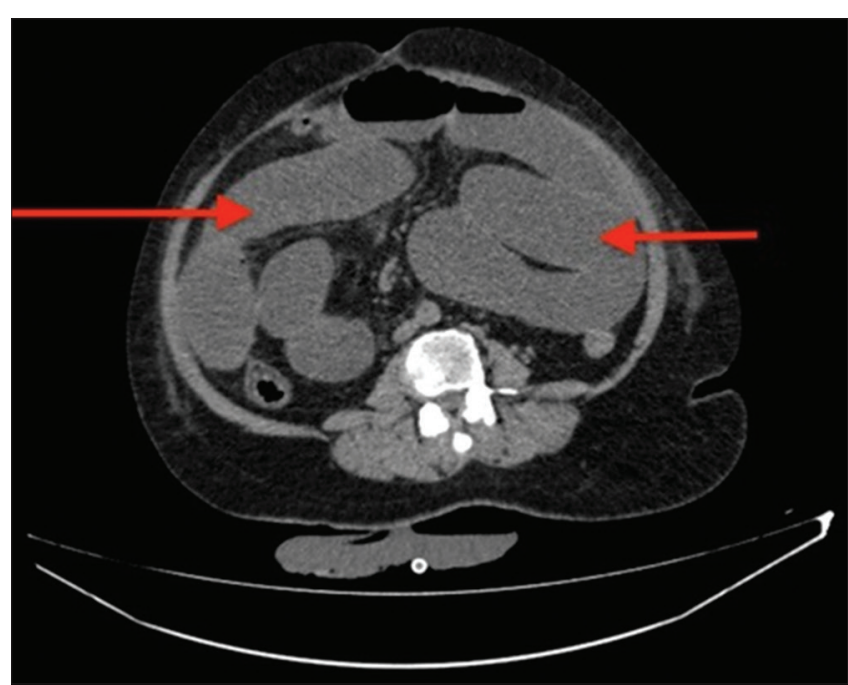

Figure 1: Computed tomography scan showing small bowel distension

After further resuscitation, she had an urgent laparotomy. The findings at surgery were grossly distended jejunum and proximal ileum, with herniation of $70 \mathrm{~cm}$ of mid-ileum through an aperture formed by two adjacent, joined appendices epiploicae of the sigmoid colon (Figure 2).

The band, thus, formed was divided into release the entrapped loop of small bowel which was found to be viable and not requiring resection. Post-operative course was uneventful and the patient was discharged on the $8^{\text {th }}$ post-operative day and now on follow-up.

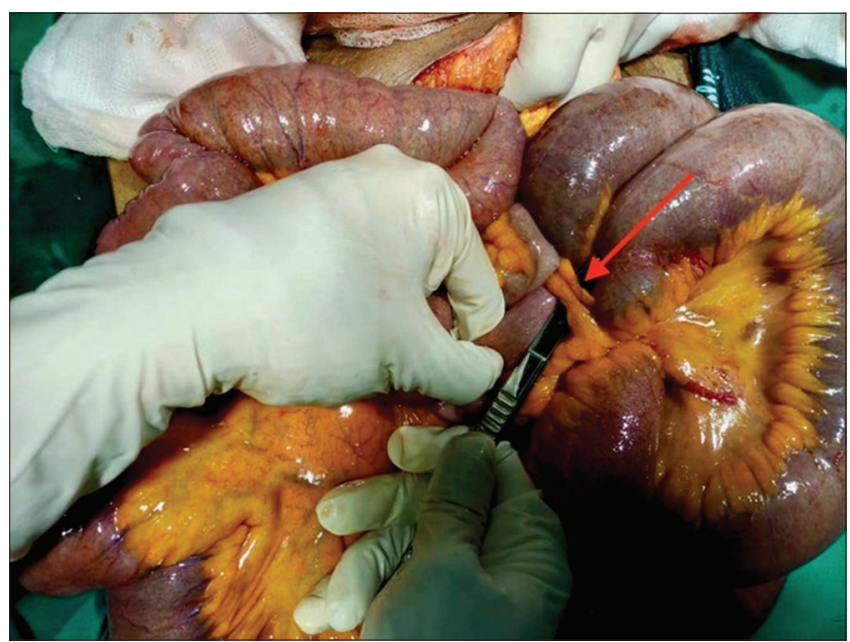

Figure 2: Distended small bowel, herniated bowel, and band reached due to the rarity of this disease entity, nonspecific presentation pattern, and limited usefulness of imaging in these cases [2], [8].

The first reported case of herniation of bowel through a defect in the appendices epiploicae was described by Lau and Ong in 1984 [3]. Krijgsman and Salter at Westcliff-On-Sea, Essex, United Kingdom, 13 years later, reported the second case of internal hernia occurring through a defect in an epiploica of the sigmoid colon through which a segment of small bowel herniated and was strangulated, which was resected [4]. There have been a total of six documented cases in the literature before our own report which is the seventh [3], [4], [5], [6], [7], [8].

There is no "typical" pattern of presentation as clinical features are non-specific [1], [2], [8]. At present, CT scan is an essential investigation in the assessment of patients presenting with intestinal obstruction, but despite its widespread use, CT scan diagnosis of internal hernia is still challenging, and diagnosis is usually made at surgery, except in few patients [1], [2]. The major concern in the management of internal hernia is that delay in diagnosis may lead to bowel gangrene and thus increased morbidity and mortality; therefore, a high index of suspicion with prompt surgical intervention will improve outcome [8]

In this patient, who had not been operated hitherto, the cause of the joining of two adjacent appendices epiploicae is not known; however, an author who described this condition presumed that it may be due to mild inflammation in the appendices [8], but this is not verifiable. Our patient also had no clinical indication of the previous primary peritonitis or pelvic inflammatory disease. Division of the joined appendices sufficed in our patient.

Laparoscopy is also valuable for both its diagnostic and minimally invasive forms of treatment [7]. Suzuki et al., in Tokyo, who reported the fifth case of internal hernia caused by epiploic appendices, diagnosed and treated the patient by single-incision laparoscopic surgery successfully [7]. However, it would not have been possible to offer our patient diagnostic and therapeutic laparoscopy despite its availability at our center due to her grossly distended and tense abdomen from late presentation due to the raised risk of bowel perforation and ensuing peritonitis.

\section{Conclusion}

Appendices epiploicae are peritoneumlined protuberances of subserosal fat along the antimesenteric taeniae coli. Two appendices can adhere to each other to create an abnormal aperture [8] Pre-operative suspicion and diagnosis are not usually
Internal hernias are a rare but important cause of intestinal obstruction and should be considered in any patient with acute intestinal obstruction without an external hernia, a prior history of intra-abdominal 
surgery, or features suggestive of obstructing intraabdominal tumor. Prompt surgical intervention is crucial to improve outcome.

\section{References}

1. Kulacoglu $H$, Tumer $H$, Aktimur $R$, Kusdemir A. Internal herniation with fatal outcome: Herniation through an unusual apertura between epiploic appendices and greater omentum. Acta Chir Belg. 2006;106(1):109-11. https://doi.org/10.1080/00 015458.2006.11679849

PMid: 16612930

2. Kar S, Mohapatra V, Rath PK. A rare type of primary internal hernia causing small intestinal obstruction. Case Rep Surg. 2016;2016:3540794. https://doi.org/10.1155/2016/3540794

PMid: 27999703

3. Lau JT, Ong GB. Bowel herniation through a defect in the appendices epiploicae. J R Coll Surg Edinb. 1984;29(3):187-8. http://dx.doi.org/10.1136/bmjgh-2019-001604
PMid: 6747927

4. Krijgsman B, Salter MC. Internal herniation through a colonic epiploica. Surgery. 1997;122(3):643. https://doi.org/10.1016/ s0039-6060(97)90142-2

PMid: 9308627

5. Liu $\mathrm{CH}$, Wang SY. Ileal herniation into a sigmoid colon epiploic fat foramen extrasigmoid hernia. Tech Coloproctol. 2004;8(2):1068. https://doi.org/10.1007/s10151-004-0066-4

PMid: 15309648

6. Oneyama M, Sekikawa K, Goto M, Kitamura M, Ohta R, Takawashi Y. A case of ileus due to an internal hernia caused by a sigmoid colon epiploic appendix. J Jpn Surg Assoc. 2011;72(12):3175-9. https://doi.org/10.3919/jjsa.72.3175

7. Suzuki $Y$, Katagiri $H$, Yoshinaga $Y$, Lefor AT, Mizokami K. Internal hernia caused by epiploic appendices successfully treated by single-incision laparoscopic surgery (SILS). Hernia. 2015;19(6):1011-3. https://doi.org/10.1007/s10029-014-1231-y PMid: 24577739

8. Srivastava KN, Agarwal A, Roopesh D, Prashad R. An unusual case of intestinal obstruction due to internal herniation from adhesions between two appendices epiploicae. J Surg Case Rep. 2018;2018(9):rjy239. https://doi.org/10.1093/jscr/rjy239 PMid: 30220991 